Pacific Journal of Mathematics

DETERMINANTAL IDEALS, IDENTITIES, AND THE 


\section{DETERMINANTAL IDEALS, IDENTITIES, AND THE WRONSKIAN}

D. G. MEAD

In this paper we examine the question raised by J. F. Ritt in his Colloquium Publication, Differential Algebra concerning the study of the differential ideals generated by the Wronskian. A test for an element to be a member of a certain (algebraic) ideal is presented and this result is applied to the differential ideal generated by the Wronskian. All identities of a certain type of determinant are also obtained.

This extends recent results concerning the Wronskian.

o. Introduction. Let $y_{i j}, i \in N=\{1,2, \cdots, n\}, j \in M=\{0,1$, $2, \cdots\}$ be indeterminates over a field $F$, and let $\mathscr{R}=F\left[y_{i j}\right]$. With $S=\left\{i_{1}, \cdots, i_{k}\right\} \subset N$ and $\left\{a_{1}, \cdots, a_{k}\right\} \subset M$, where $i_{j}<i_{j+1}$ and $a_{j}<a_{j+1}$ for all $j$, we let $\left(S ; a_{1}, \cdots, a_{k}\right)$ represent the $k \times k$ determinant with $y_{i_{p} a_{q}}$ in the $p$ th row and $q$ th column. In this notation $y_{i j}=(\{i\} ; j)$. We call $w=\sum_{j} a_{j}$ the weight of $\left(S ; a_{1}, \cdots, a_{k}\right)$. If $F$ is of characteristic zero and $y_{i j}$ is the $j$ th derivative of $y_{i}$, then $(N ; 0,1, \cdots$, $n-1)=W\left(y_{1}, \cdots, y_{n}\right)$, the Wronskian of $y_{1}, \cdots, y_{n}$. Using the Wronskian as a model we consider ideals in $\mathscr{R}$

$$
I_{t}=\left(W_{0}, W_{1}, \cdots, W_{t}\right)
$$

where $W_{i}$ is any fixed linear combination with nonzero coefficients in $F$, of all $n$th order determinants of weight $(n(n-1) / 2)+i$. We present a constructive procedure to determine whether any given $P \in \mathscr{R}$ is in $I=I_{0} \cup I_{1} \cup I_{2} \cup \cdots$ A basis of $\mathscr{R}$ as a vector space over $F$ is obtained such that the deletion of certain elements yields a basis of $\mathscr{R} / I$; this, in a natural manner, leads to cannonical forms. The proof provides all possible identities of determinants (each of the type $\left.\left(S ; a_{1}, \cdots, a_{k}\right)\right)$. We also encounter a combinatorial problem whose solution appears to be difficult.

This work extends the results of [6] to $n>2$ and those of [1] to a general ring. It has some similarities to the work of Levi [3], for the differential ideals $\left[y^{p}\right]$ and $[u v]$ as well as [2], [4], [5], and [7]. Although the Wronskian is zero if and only if $\left\{y_{i}\right\}$ is a linearly dependent set (the $y$ 's being analytic functions) [8, p. 34], by the RittRaudenbush Theorem of Zeros [8, p. 27] one cannot distinguish by zeros, elements in $\{I\}$ from those in $I$. Thus a test for membership in $I$ cannot be stated in terms of solutions. 
1. Gradings of $\mathscr{R}$. The set of all polynomials in $\mathscr{R}$ which are homogeneous of degree $d_{i}$ in the $y_{i j}$, where the $d_{i}$ are fixed nonnegative integers, is a subspace of $\mathscr{R} . \mathscr{R}$ is the direct sum of the subspaces which clearly provides a grading of $\mathscr{R}$. If $d$ is a fixed nonnegative integer and $\left\{k_{1}, \cdots, k_{d}\right\}$ is a fixed set of nonnegative integers (with possible repetitions), the set of polynomials in products of degree $d$, $y_{i_{1} k_{1}} \cdots y_{i_{d} k_{d}}$ for any choice of the $i_{j}$, is also a subspace of $\mathscr{R}$ and $\mathscr{R}$ is the direct sum of these subspaces. (With $d=0$, the subspace is $F$.) The intersection of these two gradings is the one we use, and we usually work in a subspace which is homogeneous with respect to both gradings. It is clear that each component (homogeneous subspace) is of finite dimensions. The combinatorial problem to which we referred previously is to determine the dimension of these components. Although the problem seems difficult to answer, one can obtain interesting relations by constructing different bases of a component (and then use the fact that the cardinality of all bases of the component are the same).

2. Ordering. The proofs will use an ordering of the elements in the components (of our grading). We first order subsets of $N=$ $\{1, \cdots, n\}$, then determinants, and finally products of determinants.

Definition 1. Let $S=\left\{i_{1}, \cdots, i_{k}\right\}, S^{\prime}=\left\{i_{1}^{\prime}, \cdots, i_{l}^{\prime}\right\}$ be subsets of $N$ with $i_{\alpha}<i_{\alpha+1}$ and $i_{\beta}^{\prime}<i_{\beta+1}^{\prime}$ for all $\alpha$ and $\beta$.

We say that $S$ is lower than $S^{\prime}\left(S<S^{\prime}\right)$ if

$$
k>l
$$

or

$$
k=l \text { and } \exists t \ni i_{j}=i_{j}^{\prime} \text { for } j<t \text { and } i_{t}<i_{t}^{\prime} \text {. }
$$

We also write $S \ll S^{\prime}$ if $k \geqq l$ and $i_{j} \leqq i_{j}^{\prime}$ for all $j \leqq l$. (Note that for any $S, S \ll S$ although $S \nless S$.)

Definition 2. Let $P=\left(S ; a_{1}, \cdots, a_{k}\right)$ and $P^{\prime}=\left(S^{\prime} ; b_{1}, \cdots, b_{l}\right)$ with $S$ and $S^{\prime}$ as above and $a_{\alpha}<a_{\alpha+1}$ and $b_{\beta}<b_{\beta+1}$ for all $\alpha$ and $\beta$. We say that $P$ is lower than $P^{\prime}\left(P<P^{\prime}\right)$ if

$$
S<S^{\prime}
$$

or

$$
S=S^{\prime} \text { and } \sum a_{i}<\sum b_{i}
$$


(3) $S=S^{\prime}, \sum a_{i}=\sum b_{i}$ and $\exists t \ni a_{i}=b_{i}$ for $i<t$ and $a_{t}<b_{t}$. $P$ is said to be $s$-lower than $P^{\prime}$ if either $S<S^{\prime}$ or $S=S^{\prime}$ and $\sum a_{i}<\sum b_{i}$.

If $S \ll S^{\prime}$ and $a_{j} \leqq b_{j}$ for all $j \leqq l$, we say the product $P P^{\prime}$ is monotonically increasing and we write $P \ll P^{\prime}$.

The following fact will be critical in the proof of Theorem 3: If $P$ is $s$-lower than $P^{\prime}$ and $Q$ is lower than but not $s$-lower than $P^{\prime}$ then $P$ is lower than $Q$ (in fact $s$-lower than $Q$ ).

Definition 3. Let $A=P_{1} \cdots P_{r}$ and $B=Q_{1} \cdots Q_{s}$ be products of determinants with $P_{r}$ not in $F$, and $P_{\alpha} \leqq P_{\alpha+1}, Q_{\beta} \leqq Q_{\beta+1}$ for all $\alpha$ and $\beta$. We say that $A$ is lower than $B$ if

$$
r>s
$$

or

$$
r=s \text { and } \exists t \ni P_{i}=Q_{i} \text { for } i<t \text { and } P_{t}<Q_{t} \text {. }
$$

$A$ is said to be $s$-lower than $B$ if $r>s$, or if in (2), $P_{t}$ is $s$-lower than $Q_{t}$. If $A$ is lower (s-lower) than $B$ we say that $A \cdot 1$ is lower (s-lower) than both $B$ and $B \cdot 1$.

It is clear that this is a partial ordering of $\mathscr{R}$ which is compatible with the ring multiplication; i.e. if $A<B$ then $A C<B C$ for every product $C$ of determinants, $C \neq 0$.

Definition 4. If the product $A=P_{1} \cdots P_{r}=\sum \alpha_{i} B_{i}$ with $\alpha_{i} \in F$, then $A$ is said to be replaceable ( $s$-replaceable) if for every $i, B_{i}$ is lower (s-lower) than $A$.

3. Basis. We now consider certain products, called $\lambda$-products, the set of which form a basis of $\mathscr{R}$ (as a vector space over $F$ ).

Definition 5. The product $A$, which can be written $A=P_{1} \cdots P_{r}$, is called a $\lambda$-product if $A=1$ or $r>0, P_{r} \notin F$, and if $i<j$ then $P_{i} \ll P_{j}$.

We first show the $\lambda$-products span $\mathscr{R}$.

Lemma 1. If a product $A$ is not a $\lambda$-product, then $A$ is $s$ replaceable.

Proof. Let $P=\left(\left\{i_{1}, \cdots, i_{k}\right\} ; a_{1}, \cdots, a_{k}\right)<P^{\prime}=\left(\left\{i_{1}^{\prime}, \cdots, i_{l}^{\prime}\right\} ; b_{1}, \cdots\right.$, $\left.b_{l}\right)$ but not $P \ll P^{\prime}$. We consider two cases:

$$
i_{j} \leqq i_{j}^{\prime} \text { for } j<t \text { but } i_{t}>i_{t}^{\prime} \text {. }
$$




$$
S \ll S^{\prime}, a_{j} \leqq b_{j} \text { for } j \leqq t \text { but } a_{t}>b_{t} .
$$

Since $P<P^{\prime}, k \geqq l$. Assume (1) is satisfied and consider the $(k+l) \times(k+l)$ matrix

$$
H=\left(\begin{array}{ll}
B_{1} & C \\
D & B_{2}
\end{array}\right)
$$

where $B_{1}$ is the $k \times k$ matrix of $P, B_{2}$ ist he $l \times l$ matrix of $P^{\prime}, C=$ $\left(c_{p, q}\right)$ is the $k \times l$ matrix defined by $c_{p, q}=\left\{\begin{array}{l}0 \text { if } p<t \\ y_{i_{p} b_{q}} \text { if } p \geqq t,\end{array}\right.$ and $D=\left(d_{p q}\right)$ is the $l \times k$ matrix defined by $d_{p, q}=\left\{\begin{array}{l}y_{i^{\prime}{ }_{p q} a_{q}} \text { if } p \leqq t . \\ 0 \text { if } p>t .\end{array}\right.$

Expand $\operatorname{det}(H)$ in two ways: by minors of the first $k$ columns, and by minors of $l-1$ rows consisting of the first $t-1$ rows along with the last $l-t$ rows. These two expansions give this same value $(\operatorname{det} H)$; set them equal to each other and solve for $P \cdot P^{\prime}=$ $\left(\operatorname{det} B_{1}\right)\left(\operatorname{det} B_{2}\right)$, which appears in the first expansion. We claim that all the other terms are $s$-lower that $P \cdot P^{\prime}$.

Clearly all the terms in the row-expansion are $s$-lower, since one of the two determinants in each term is a $(k+1)$ st order determinant, hence the term is $s$-lower than $P P^{\prime}$.

In the column expansion we see the $k$ th order determinant is of the form $\left(\left\{i_{1}, \cdots, i_{t-1}, e_{1}, \cdots, e_{k-t+1}\right\} ;-\right)$ where $\left\{e_{1}, \cdots, e_{k-t+1}\right\}$ runs over all possible subsets of $\left\{i_{1}^{\prime}, \cdots, i_{t}^{\prime}, i_{t}, \cdots, i_{k}\right\}$. Since this latter subset is monotonically increasing as written, we see that the highest $k$ th order determinant is obtained when $\left\{e_{1}, \cdots, e_{k-t+1}\right\}=\left\{i_{t}, \cdots, i_{k}\right\}$, that is, when the determinant is $P$. Thus all the other terms in the column expansion are lower, and clearly $s$-lower than $P P^{\prime}$. This completes the proof when (1) is satisfied.

The proof for the case in which (2) is satisfied is similar. With the notation as above, consider the $(k+l) \times(k+l)$ matrix

$$
H_{1}=\left(\begin{array}{ll}
B_{1} & E \\
F & B_{2}
\end{array}\right) \text { where } e_{p q}=\left\{\begin{array}{ll}
y_{i_{p} b_{q}} & \text { if } q \leqq t \\
0 & \text { if } q>t
\end{array} \text { and } f_{p q}= \begin{cases}0 & \text { if } q<t \\
y_{i_{p}^{\prime} a_{q}} & \text { if } q \geqq t .\end{cases}\right.
$$

Expand $\operatorname{det}\left(H_{1}\right)$ by minors of the first $k$ rows, and by minors of $l-1$ columns consisting of the first $t-1$ columns along with the last $l-t$ columns. The highest product in the two expansions is $P_{1} P_{2}$ and all the other terms as $s$-lower.

The process indicated in Lemma 1 , replacing a product which is not a $\lambda$-product, always takes place inside one of the (homogeneous) components of our grading, and since the dimension of this subspace is finite, the process must terminate. We have proved

THEOREM 1. The $\lambda$-products span $\mathscr{R}$ (as a vector space over $F$ ). 
We next show that the set of $\lambda$-products is linearly independent over $F$. This will show we have obtained all of the determinantal identities, for, by the independence of the $\lambda$-products, the representation of any element of $\mathscr{R}$ as a linear combination of $\lambda$-products must be unique.

THEOREM 2. The set of $\lambda$-products is linearly independent over $F$.

Proof. We proceed by induction on $n$ (the cardinality of the set of the first subscripts on the $y$ 's). The theorem is clearly true if $n=0$ or 1 .

We extract an essential step in the induction proof as

Lemma 2. If (1) $\sum \alpha_{i} L_{i}=0, i \in I$ with the $L_{i}$ 's distinct $\lambda$-products and $\alpha_{i} \in F$, then there exists a nonempty subset $J \subset I$ and $a$ set of distinct $\lambda$-products $L_{j}^{\prime}, j \in J$ such that

$$
\sum \alpha_{j} L_{j}^{\prime}=0, j \in J
$$

with $y_{n k}$ appearing in $L_{j}^{\prime}$ only in nth order determinants (i.e. determinants $(N ; \ldots))$.

Let us use Lemma 2 to complete the proof of Theorem 2, and then return to prove the lemma. Since $(*)$ is an identity in the $y_{i j}$, we may assume each $L_{j}^{\prime}$ is of the same degree, say $k$, in $n$th order determinants. Then $L_{j}^{\prime}$ has a factor $\left(N ; a_{1}(j), \cdots, a_{n}(j)\right) \cdots\left(N ; k_{1}(j), \cdots\right.$, $\left.k_{n}(j)\right)$ with $a_{n}(j) \leqq b_{n}(j) \leqq \cdots \leqq k_{n}(j)$. We order the sets $T_{j}=\left(a_{n}(j), \cdots\right.$, $k_{n}(j)$ ) lexicographically, and let $J^{\prime}=\left\{j \in J \mid T_{j}\right.$ is highest $\}$. Again, since $(*)$ is an identity, we must have $\sum \alpha_{j} L_{j}^{\prime}=0$ for $j \in J^{\prime}$. In this last equation, if $\left(t_{1}, \cdots, t_{k}\right)$ is the highest $T_{j}$, then the coefficient of $\prod_{i=1}^{k} y_{n t_{i}}$ is $\sum \alpha_{j} L_{j}^{\prime \prime}=0$ for $j \in J^{\prime}$, where $L_{j}^{\prime \prime}$ is obtained from $L_{j}^{\prime}$ by replacing each $\left(N ; a_{1}, \cdots, a_{n}\right)$ by $\left(\{1, \cdots, n-1\} ; a_{1}, \cdots, a_{n-1}\right)$. We note that the $L_{j}^{\prime \prime}$ are distinct $\lambda$-products free of $y_{n}$; by the induction hypothesis each $\alpha_{j}=0$. This completes the proof of Theorem 2 .

We return to the proof of Lemma 2, first making some observations concerning the behavior of $\lambda$-products under a particular type of substitution.

If $L$ is a fixed $\lambda$-product, then substitution of $y_{2 j}+t y_{1 j}$ for $y_{2 j}$ will turn $L$ into a polynomial in $t$ of degree, say, $d$. The coefficient of $t^{d}$ is again a $\lambda$-product $L^{\prime}$ and the only $y_{2 j}$ which remain in $L^{\prime}$ are those in determinants $(\{1,2, \cdots,\} ; \ldots)$. Knowing $d$ and $L^{\prime}$, we can reconstruct $L$; i.e. the function from $(L, d)$ for certain $L$ to $L^{\prime}$ is one-one. An example will illustrate the procedure. If $L=(\{1,2,4\}$; 
$\ldots)(\{1,3,4\} ; \ldots)(\{2,3\} ; \ldots)(\{2\} ; \ldots)(\{3\} ; \ldots)$ then $d=2$ and $L^{\prime}=(\{1,2,4\}$; $\ldots)(\{1,3,4\} ; \ldots)(\{1,3\} ; \ldots)(\{1\}$;__ $)(\{3\} ; \ldots)$. (There is no change in the second subscripts.) To construct $L$ from $L^{\prime}$, we note that since $d=2$, two of the 1's in $L^{\prime}$ need to be changed to 2's. From the definition of a $\lambda$-product, it is clear that we change the 1 's to 2's, starting from the right (if the determinants in the $\lambda$-product are arranged so they are monotonically increasing). This procedure can easily be generalized.

In (1) $\sum \alpha_{i} L_{i}=0$ with the $L_{i}$ distinct $\lambda$-products we make the substitution $y_{2 j}+t y_{1 j}$ for $y_{2 j}$ and extract the coefficient of $t^{d}$ where $d=\max \left(d_{i}\right), L_{i}$ having become a polynomial of degree $d_{i}$ in $t$. Since (1) was an identity in the $y_{i j}$, it follows that the coefficient of $t^{d}$ must be zero. The function from $(L, d)$ for certain $L$ to $L^{\prime}$ is one-one, therefore

$$
\sum \alpha_{i} L_{i}^{\prime}=0 \text { for } i \in I^{\prime} \leqq I,
$$

where the $L_{i}^{\prime}$ are distinct $\lambda$-products and the only 2's that appear as a first subscript are in determinants of order two or higher of the form $(\{1,2, \cdots\} ; \ldots)$.

Continue the process, substituting $y_{3 j}=y_{3 j}+t y_{2 j}$ and again extract the coefficient of the highest power of $t$. We find

$$
\sum \alpha_{i} L_{i}^{\prime \prime}=0 \text { for } i \in I^{\prime \prime} \subset I^{\prime},
$$

where the $L_{i}^{\prime \prime}$ are distinct $\lambda$-products and the only 3's that appear as a first subscript are in determinants of order three on higher $(\{1,2,3, \cdots,\} ; \ldots)$. The proof of the lemma is completed by induction.

Combining Theorems 1 and 2, we note that the $\lambda$-terms form a basis for $\mathscr{R}$ as a vector spaces over $F$. In the next section we consider a subset of the $\lambda$-products which form a basis of $\mathscr{R} / I$, and this will yield canonical forms and the test we seek for membership in $I$.

4. Canonical forms. With $r>1$ and $w \geqq(r(r-1)) / 2$, let

$$
M(r, w)=\left\{a_{1}, \cdots, a_{r}\right\} \text { where } w=\sum a_{i}
$$

and either

$$
a_{i+1}=1+a_{i} \text { for all } i
$$

or

$$
a_{i+1}=1+a_{i} \text { for } i \neq t \text { for some } t \text { and } a_{t+1}=2+a_{t} \text {. }
$$

Definition 6. Let $P$ be a $\lambda$-product, $P \neq 1$. Then $P$ is called a $\beta$-product if $P$ contains no subproduct (i.e. factor) of one of the 
following types :

(1) $C=\left(N ; c_{1}, \cdots, c_{n}\right)$ and $\left\{c_{1}, \cdots, c_{n}\right\}=M(n, w)$ for some $w$.

(2) $B \cdot C=\left(N ; b_{1}, \cdots, b_{n}\right)\left(N ; c_{1}, \cdots, c_{n}\right)$ where $B \ll C$,

$b_{i}=c_{i}$ for $i>t$ and $\left\{c_{1}, \cdots, c_{t}\right\}=M(t, w)$ for some $w$.

(3) $C \cdot D=\left(N ; c_{1}, \cdots, c_{n}\right)\left(S ; d_{1}, \cdots, d_{k}\right)$ where $C \ll D$, $c_{i}=d_{i}$ for $i \leqq r$ and $\left\{c_{r+1}, \cdots, c_{n}\right\}=M(n-r, w)$ for some $w$.

(4) $B \cdot C \cdot D=\left(N ; b_{1}, \cdots, b_{n}\right)\left(N ; c_{1}, \cdots, c_{n}\right)\left(S ; d_{1}, \cdots, d_{k}\right)$ where $B \ll C \ll D, b_{i}=c_{i}$ for $i>t, c_{i}=d_{i}$ for $i \leqq r$, and $\left\{c_{r+1}, \cdots, c_{t}\right\}=M(t-r, w)$ for some $w$.

Since $P=P \cdot 1$ we consider $P$ to be a subproduct of itself.

Although all the conditions can be obtained as special cases of (4), taking one or both of $B$ and $D$ to be 1 , it seems worthwhile to indicate the various cases explicitly.

An alternate characterization of $\beta$-products, contained in the next lemma, is used in the proofs of the two following theorems.

LEMma 3. Let $P$ be a fixed $\lambda$-product and $w$ a positive integer no less than $n(n-1) / 2$. If $E$ is an nth order determinant of weight $w$, then for $P E$ to be a $\lambda$-product but not a $\beta$-product with $E$ playing the role of $C$, it is necessary and sufficient that $P E$ be the highest $\lambda$-product in the set $\{P X\}$ where $X$ runs over nth order determinants of weight $w$.

Proof. It is clear that we may consider only the last condition of Definition 6 and assume $P E=B D E$ with $B \ll E \ll D$. For the necessity we assume $B D E$ is not a $\beta$-product with $E$ playing the role of $C$, that there is an $n$th order $F$ of weight $w$ such that $B D F$ is a $\lambda$-product higher than $B D E$, and we obtain a contradiction. We know that $F=\left(N ; f_{1}, \cdots, f_{n}\right)$ is higher than $E=\left(N ; e_{1}, \cdots, e_{n}\right)$; of course $B \ll F \ll D$. Let $e_{i}=b_{i}$ for $i>t, e_{i}=d_{i}$ for $i \leqq r$, and $\left\{e_{n+1}, \cdots, e_{t}\right\}=$ $M\left(t-r, w^{\prime}\right)$ for some $w^{\prime}$. Since $E \ll F$ there is an $s$ such that $f_{i}=e_{i}$ for $i<s$ and $f_{s}>e_{s}$. Clearly $s>r$ since $f_{i}=d_{i}=e_{i}$ for $i \leqq r$. If $r<s \leqq t$ then $\sum_{i=r+1}^{t} f_{i}>\sum_{i=r+1}^{t} e_{i}(M(k, w)$ is the highest $k$-tuple of weight $w$ ), hence $f_{j}<e_{j}$ for some $j>t$. But this is a contradiction since $f_{i} \geqq b_{i}$ for all $i$ and $b_{i}=e_{i}$ for $i>t$. Similarly $s$ cannot be larger than $t$. Consequently there can be no $s$ with $f_{s}>e_{s}$ and this completes the proof of the necessity.

The proof of the sufficiency is direct and we assume that $B D E$ is the highest $\lambda$-product of the form $B D X$ where $X$ is an $n$th order determinant of weight $w$. If $E=D$ and we are done for $E$ would play the role of $C$ in Condition 3. Assume $E \neq D$, let $q$ be such 
that $e_{i}=d_{i}$ for $i<q$ and $e_{q}<d_{q}$ ( $q$ is the order of $D$ if $e_{i}=d_{i}$ for every $i$ ), and let $s$ be the smallest $i \geqq q$ such that $e_{i+1}>$ $1+e_{i}$. Clearly $1+e_{s} \leqq d_{s}$. If there is an $l>s+1$ such that $e_{l-1}<$ $e_{l}-1$, let $F=\left(N ; f_{1}, \cdots, f_{n}\right)$ where $f_{i}=e_{i}$ for $i \neq s, l, f_{s}=1+e_{s}$ and $f_{l}=e_{l}-1$. Then the weight of $F$ is $w$ and $B D F$, being higher than $B D E$, is not a $\lambda$-product; this implies that $f_{l}<b_{l}=e_{l}$. Consequently, for any $j \geqq i>s+1$ with $e_{i}>1+e_{i-1}$ we have $e_{j}=b_{j}$. (In the same way, if $e_{s+1}-e_{s}>2$ then $e_{j}=b_{j}$ for $j \geqq s+1$.) If $e_{s+1}=$ $2+e_{s}$ and $l$ is the smallest $i>s+1$ such that $e_{i}>1+e_{i-1}$ then $E$ plays the role of $C$ in Condition 4 with $t=l-1$ and $r=q-1$. If $e_{s+1}>2+e_{s}$ then $E$ plays the role of the same $C$ with $t=s$ and $r=q-1$. This completes the proof of the lemma.

Theorem 3. If the $\lambda$-product $P$ is not a $\beta$-product, then $P$ is replaceable modulo $I$.

Proof. If $P$ is not a $\beta$-product, then $P$ contains a subproduct of one of the four types described in the definition. Pick one of these subproducts and let $C$ denote the corresponding $n$th order determinant. Since $C$ is one of the terms in $W_{k}$, where $k=\sum_{i=1}^{n} c_{i}-n(n-1) / 2$, we can solve $(P / C) W_{k} \equiv 0(\bmod I)$ for $P$. By the necessity part of Lemma 3 , we see that every $\lambda$-product in the expression we have obtained for $P$ is lower than $P$. If a product, $R$, in the expression for $P$ is not a $\lambda$-product, we know $R$ is $s$-replaceable (Lemma 1 ). If $P$ is lower than $R$ it is not $s$-lower than $R$, hence every product in the representation of $R$ as a linear combination $s$-lower products is lower than $P$ (see the remark after definition 2). This completes the proof of Theorem 3 .

In this theorem the products are not contained in one component of our grading. However, the total weight provides a grading and if we consider the grading which is the intersection of the total weight with the degree $\left(d_{i}\right.$ in the $\left.y_{i j}\right)$, then all of the products above will be in one homogeneous component of this new grading. Since the dimension of each of these components is finite, we may conclude that every $\lambda$-product is congruent modulo $I$ to a sum of $\beta$-products.

THEOREM 4. (Canonical forms). Every element of $\mathscr{R}$ is expressible as a linear combination, with coefficients in $F$, of a finite number of distinct products

$P W_{a} W_{b} \cdots W_{r}$

where $P$ is a $\beta$-product or 1 . The expression, which may be of degree zero in the W's, is unique. 
Proof. The fact that such a representation can be obtained follows from Theorem 3, and we merely need to show uniqueness. For each expression $T$ of the form $(*)$ we will obtain the highest $\lambda$-product $B$ in the expression for $T$ as a linear combination of $\lambda$-products. The correspondence $T \rightarrow B$ is one-to-one, hence no linear combination of distinct expressions $T$ of the form $(*)$ can vanish since the highest $B$ cannot cancel.

If $E$ and $F$ are $n$th order determinants of weight $w, P$ is a $\lambda$ product, $P E$ is the highest $\lambda$-product of the form $P$ times an $n$th order determinant of weight $w, P F$ is not a $\lambda$-product, and $P F=\sum Q_{i}$ where the $Q_{i}$ are $\lambda$-products, then, by the remark after Definition 2, each $Q_{i}$ is $s$-lower than $P F$ and therefore $s$-lower than $P E$. In view of the above, we see that the desired correspondence is provided by the sufficiency part of Lemma 3, and this completes the proof of the theorem.

Corollary 1. The $\beta$-product form a basis of $\mathscr{R} \bmod I$.

CoRollary 2. A necessary and sufficient condition for an element of $\mathscr{R}$ to be in $I$ is that none of the terms $(*)$ of its canonical form be of degree zero in the W's.

One can obtain many facts similar to those obtained in [6], such as the following

Corollary 3. Let $P$ be a $\beta$-product of degree $d$ in nth order determinants and degree $t$ in $(n-1)$ st order determinants

$$
\left.P=\prod_{i=1}^{d}\left(N ; a_{1 i}, \cdots, a_{n i}\right) \prod_{i=1}^{t} S_{i} ; b_{1 i}, \cdots, b_{n-1 i}\right)
$$

then

$$
\begin{gathered}
\sum_{j=1}^{n} \sum_{i=1}^{d} a_{j i}+\sum_{j=1}^{n-1} \sum_{i=1}^{t} b_{j i} \geqq d^{2}+d\left(\frac{n^{2}-n+2}{2}\right) \\
+t\left(\frac{(n-3)(n-2)}{2}+n+d-2\right) .
\end{gathered}
$$

Proof. The $\beta$-product of minimal weight with the proper degrees is

$$
\begin{array}{r}
\left(\prod_{i=1}^{d}(N ; 0,1, \cdots, n-3, n-3+i, n+i)\right) \\
\quad \times\left(\prod_{i=1}^{t}\left(S_{i} ; 0,1, \cdots, n-3, n+d-2\right)\right)
\end{array}
$$


where the cardinality of each $S_{i}$ is $n-1$.

We conclude with a combinatorial result. Let $d_{1}, d_{2}$, and $d_{3}$ be three positive integers and $S$ a fixed set of non-negative integers, (possibly with repetitions) where $|S|=d_{1}+d_{2}+d_{3}$. (We use $|S|$ to denote the cardinality of $S$.) Let $K$ be the number of distinct ways of writing $S$ as the union of three subsets, $A_{1}, A_{2}$, and $A_{3}$ where $\left|A_{i}\right|=d_{i}$. We also consider $S$ as the union of six special kinds of subsets and show that the number of ways of doing this is also $K$. Before describing these subsets, we define some terms relating to subsets of integers.

A set $A$ of ordered $n$-tuples is said to contain strict $n$-tuples if $U \in A$ implies $U=\left\{u_{1}, \cdots, u_{n}\right\}$ where $u_{i}<u_{i+1}$.

A set $A$ containing strict $n$-tuples is monotonic if given two elements of $A$, they can be labelled $U=\left\{u_{i}\right\}$ and $V=\left\{v_{i}\right\}$ so that $u_{i} \leqq v_{i}$ for $i=1, \cdots, n$.

The pair $A, B$, with $A$ containing strict $n$-tuples, and $B$ containing strict $m$-tuples $(A \neq B)$, is $A-B$ monotonic if given $U=\left\{u_{i}\right\} \in A$ and $V=\left\{v_{i}\right\} \in B$ then $u_{i} \leqq v_{i}$ for all $i \leqq \min (m, n)$.

Let $L$ be the number of ways that $S$ can be written as a union of six sets of subsets $A_{1}, \cdots, A_{6}$, containing strict $n_{i}$-tuples, respectively; each $A_{i}$ is monotonic and each pair $A_{i}, A_{j}$ is $A_{i}-A_{j}$ monotonic if $i<j ; n_{1}=3, n_{2}=n_{3}=2, n_{5}=n_{6}=1$ and either $n_{4}=2$ and

$$
d_{1}=\left|A_{1}\right|+\left|A_{2}\right|+\left|A_{3}\right|, d_{2}=\left|A_{1}\right|+\left|A_{2}\right|+\left|A_{4}\right|+\left|A_{5}\right|
$$

and

$$
d_{3}=\left|A_{1}\right|+\left|A_{3}\right|+\left|A_{4}\right|+\left|A_{6}\right|,
$$

or $n_{4}=1$ and

$$
d_{1}=\left|A_{1}\right|+\left|A_{2}\right|+\left|A_{3}\right|+\left|A_{4}\right|, d_{2}=\left|A_{1}\right|+\left|A_{2}\right|+\left|A_{5}\right|,
$$

and

$$
d_{3}=\left|A_{1}\right|+\left|A_{3}\right|+\left|A_{3}\right| \text {. }
$$

TheOREM $5 . \quad K=L$.

Proof. In $\mathscr{R}$ (with $n=3$ ) we consider the (homogeneous) component containing those products of degree $d_{i}$ in $y_{i}, i=1,2,3$, for which the set of the second subscripts is $S$. Since the $y_{i j}$ are linearly independent, the dimensions of this subspace is $K$.

The set $S$ can be considered that a set of $\lambda$-products and for a fixed $\lambda$-product, $A_{i}$ is the collection of all determinants $\left(S_{i} ; \ldots\right)$ where $\left|S_{i}\right|=n_{i}$. We have $S_{1}=\{1,2,3\}, S_{2}=\{1,2\}, S_{3}=\{1,3\}, S_{5}=\{2\}$, and 
$S_{6}=\{3\} . \quad$ In the first case $\left(n_{4}=2\right) S_{4}=\{2,3\}$, while in the other $\left(n_{4}=1\right) S_{4}=\{1\} . \quad$ By Theorems 1 and 2, the $\lambda$-products form a basis of the subspace and therefore the dimension of the subspace is $L$. That is, $K=L$.

This result (and its generalization) was useful in ruling out various candidates for the set of $\lambda$-products. With $|S|$ small one can determine $K$.

It would be interesting to know if there is any simple or even iterative method for computing the number $K$. Of course one can produce other sets of bases for the components, but none that we have obtained has simplified the determination of $K$.

\section{REFERENCES}

1. A. P. Hillman, D. W. Forslund and G. J. Giaccai, Determinantal ideals with applications to differential algebra, The University of New Mexico Technical Report, No. 143, August 1967.

2. A. P. Hillman, D. G. Mead, K. B. O'Keefe and E. S. O'Keefe, Ideals generated by products, Proc. Amer. Math. Soc., 17 (1966), 717-719.

3. H. Levi, On the structure of differential polynomials and on their theory of ideals, Trans. Amer. Math. Soc., 51 (1942), 532-568.

4. D. G. Mead, Differential ideals, Proc. Amer. Math. Soc., 6 (1955), 420-432.

5. - A necessary and sufficient condition for membership in [uv], Proc. Amer. Math. Soc., 17 (1966), 470-473.

6. D. G. Mead, and B. D. McLemore, Ritt's question on the Wronskian, Pacific J. Math., 35 (1970), 467-472.

7. K. B. O'Keefe and E. S. O'Keefe, The differential ideal [uv], Proc. Amer. Math. Soc. 17 (1966), 750-757.

8. J. F. Ritt, Differential Algebra, Amer. Math. Soc. Colloquium Publications, vol. 33, New York, 1950.

Received March 25, 1971.

University of CALifornia, Davis 



\section{PACIFIC JOURNAL OF MATHEMATICS}

\section{EDITORS}

H. SAMELSON

Stanford University

Stanford, California 94305

C. R. HobBY

University of Washington

Seattle, Washington 98105
J. DUGUNDJI

Department of Mathematics

University of Southern California

Los Angeles, California 90007

RICHARD ARENS

University of California

Los Angeles, California 90024

\section{ASSOCIATE EDITORS}

E. F. BECKENBACH

B. H. NeumanN

F WoLF

K. YoshidA

\section{SUPPORTING INSTITUTIONS}

UNIVERSITY OF BRITISH COLUMBIA

UNIVERSITY OF SOUTHERN CALIFORNIA

CALIFORNIA INSTITUTE OF TECHNOLOGY

UNIVERSITY OF CALIFORNIA

MONTANA STATE UNIVERSITY

STANFORD UNIVERSITY

UNIVERSITY OF NEVADA

NEW MEXICO STATE UNIVERSITY

OREGON STATE UNIVERSITY

UNIVERSITY OF OREGON

OSAKA UNIVERSITY

UNIVERSITY OF TOKYO

UNIVERSITY OF UTAH

WASHINGTON STATE UNIVERSITY

UNIVERSITY OF WASHINGTON

$\stackrel{*}{*} \stackrel{*}{*} \stackrel{*}{*}{ }^{*}{ }^{*}$ MMEICAN MATHEMATICAL SOCIETY

NAVAL WEAPONS CENTER

Printed in Japan by International Academic Printing Co., Ltd., Tokyo, Japan 


\section{Pacific Journal of Mathematics}

\section{Vol. 42, No. $1 \quad$ January, 1972}

Tage Bai Andersen, On Banach space valued extensions from split faces ........

David Marion Arnold, A duality for quotient divisible abelian groups of finite

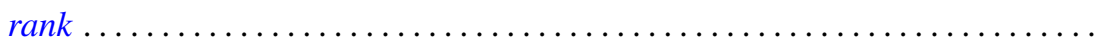

Donald Pollard Ballou, Shock sets for first order nonlinear hyperbolic

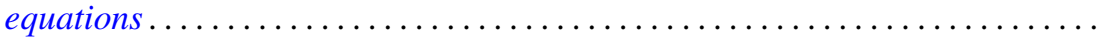

Leon Brown and Lowell J. Hansen, On the range sets of $H^{p}$ functions .........

Alexander Munro Davie and Arne Stray, Interpolation sets for analytic

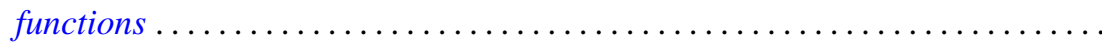

M. G. Deshpande, Structure of right subdirectly irreducible rings. II . . . . . . . . .

Barry J. Gardner, Some closure properties for torsion classes of abelian

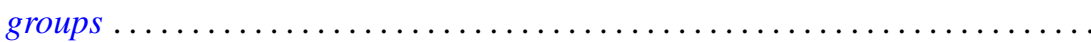

Paul Daniel Hill, Primary groups whose subgroups of smaller cardinality are

direct sums of cyclic groups . . . . . . . . . . . . . . . . . . .

Richard Allan Holzsager, When certain natural maps are equivalences .........

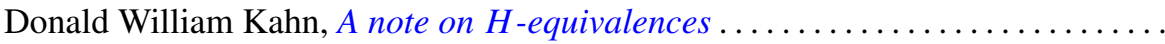

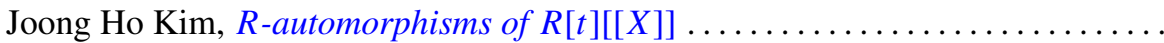

Shin'ichi Kinoshita, On elementary ideals of polyhedra in the 3-sphere.........

Andrew T. Kitchen, Watts cohomology and separability...

Vadim Komkov, A technique for the detection of oscillation of second order

ordinary differential equations .

Charles Philip Lanski and Susan Montgomery, Lie structure of prime rings of characteristic 2

Andrew Lenard, Some remarks on large Toeplitz determinants . .

Kathleen B. Levitz, A characterization of general Z.P.I.-rings. II .

Donald A. Lutz, On the reduction of rank of linear differential systems

David G. Mead, Determinantal ideals, identities, and the Wronskian ...

Arunava Mukherjea, A remark on Tonelli's theorem on integration in product

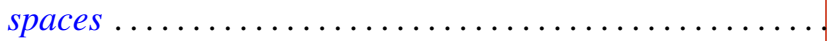

Hyo Chul Myung, A generalization of the prime radical in nonassociative rings.

John Piepenbrink, Rellich densities and an application to unconditionally nonoscillatory elliptic equations.

Michael J. Powers, Lefschetz fixed point theorems for a new class of multi-valued maps .

Aribindi Satyanarayan Rao, On the absolute matrix summability of a Fourier

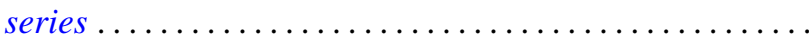

T. S. Ravisankar, On Malcev algebras ......................... 227

William Henry Ruckle, Topologies on sequences spaces . . . . . . . . . . . . . 235

Robert C. Shock, Polynomial rings over finite dimensional rings . . . . . . . . . 251

Richard Tangeman, Strong heredity in radical classes . . . . . . . . . . . . . . 259

B. R. Wenner, Finite-dimensional properties of infinite-dimensional spaces . . . . 267 